\title{
Corrigendum to: Molecular Detection of Isoniazid and Rifampin Resistance in Mycobacterium tuberculosis Isolates from Lorestan Province, Iran from 2014 to 2017
}

\author{
Shahin Najar-Peerayeh ${ }^{1, *}$ \\ ${ }^{1}$ Department of Bacteriology, Faculty of Medical Sciences, Tarbiat Modares University, Tehran, Iran \\ "Corresponding author: Department of Bacteriology, Faculty of Medical Sciences, Tarbiat Modares University, Tehran, Iran. Email: najarp_s@modares.ac.ir
}

Received 2020 April 22; Accepted 2020 April 22.

This corrects the article "Molecular Detection of Isoniazid and Rifampin Resistance in Mycobacterium tuberculosis Isolates from Lorestan Province, Iran from 2014 to 2017".

\section{Dear Editor-in-Chief,}

Due to an internal discordance between the authors of the article, we have forgotten to write the name of Ashraf Mohebati-Mobarez as one of the authors. Although the new author had already all criteria of authorship, we sincerely apologize to our readers. All authors were agreed to the below changes. Therefore, the list of authors and authors' contribution change to:

New Authors:

1. Fariborz Heydari (Heydari.fariborz@gmail.com)

Department of Bacteriology, Faculty of Medical Sciences, Tarbiat Modares University, Tehran, Iran

2. Shahin Najar-Peerayeh (najarp_s@modares.ac.ir)

Department of Bacteriology, Faculty of Medical Sciences, Tarbiat Modares University, Tehran, Iran

3. Ashraf Mohebati-Mobarez (mmmobarez@modares.ac.ir)

Department of Bacteriology, Faculty of Medical Sciences, Tarbiat Modares University, Tehran, Iran

4. Hamed Esmaeil Lashgarian

Similar to the first published version

5. Maryam Karkhane

Similar to the first published version

Authors' Contribution:

Fariborz Heidary performed laboratory experiments, Shahin Najar Peerayeh was the thesis supervisor, Ashraf Mohebati-Mobarez was the thesis advisor professor, Hamed Esmaeil Lashgarian and Maryam Karkhane edited the manuscript.

Kind Regards,

Dr. Shahin Najar-Peerayeh

Ticket: 417447, 5-April-2020 